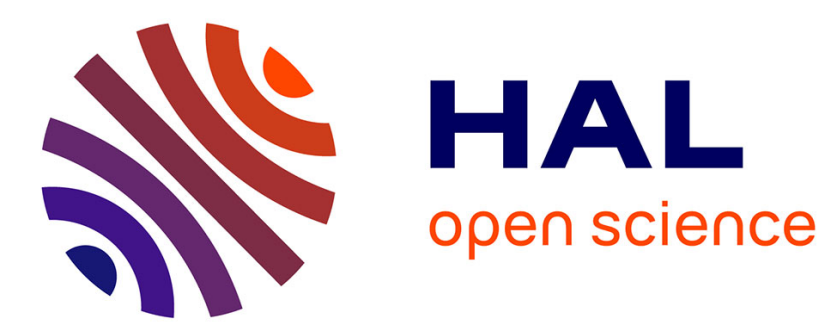

\title{
Pratiques paysannes d'exploitation des arbres et paysages forestiers du Haut Atlas marocain Jean-Brice Cordier, D. Genin
}

\section{To cite this version:}

Jean-Brice Cordier, D. Genin. Pratiques paysannes d'exploitation des arbres et paysages forestiers du Haut Atlas marocain. Revue forestière française, 2008, 60 (5), pp.571-588. 10.4267/2042/28072 . hal-03449560

\section{HAL Id: hal-03449560 https://hal.science/hal-03449560}

Submitted on 25 Nov 2021

HAL is a multi-disciplinary open access archive for the deposit and dissemination of scientific research documents, whether they are published or not. The documents may come from teaching and research institutions in France or abroad, or from public or private research centers.
L'archive ouverte pluridisciplinaire HAL, est destinée au dépôt et à la diffusion de documents scientifiques de niveau recherche, publiés ou non, émanant des établissements d'enseignement et de recherche français ou étrangers, des laboratoires publics ou privés. 


\title{
LA mULTIFONCTIONNALITE DES PAYSAgES
}

\section{Pratiques paysannes d'exploitation des arbRes ET PAYSAGES FORESTIERS DU HAUT ATLAS MAROCAIN}

\author{
Jean-Brice Cordier - Didier Genin
}

Dans le Haut Atlas marocain, la forêt constitue toujours un support important de ressources diversifiées nécessaires à la reproduction des sociétés rurales berbères qui s’y développent. En effet, dans un milieu caractérisé par de fortes pentes, un climat montagnard rigoureux en hiver et par un certain isolement géographique et de développement, les espaces forestiers permettent la collecte de bois de feu ou de construction nécessaires à la vie quotidienne des populations. Ils représentent aussi une ressource fourragère d'appoint fondamentale pour la conduite des troupeaux qui sont la base traditionnelle des systèmes de production locaux (Genin et al., 2009a). Mais, devant la relative fragilité des écosystèmes en présence (Alifriqui, 1993) et la pression anthropique importante exercée par une population humaine en croissance continue, la forêt du Haut Atlas marocain est souvent caractérisée par des signes de dégradation se manifestant par des pertes de recouvrement et de biodiversité (Gauquelin et al., 2000 ; Genin et al., 2009b). Néanmoins, les sociétés berbères ont mis en place des formes de contrôle communautaire de gestion de ces ressources en instaurant des règles d'usages des produits de la forêt comme c'est notamment le cas dans les agdals forestiers. Le terme d'origine berbère agdal ou agùdal désigne un mode d'appropriation et de gestion de la terre en bien commun mis en œuvre par une institution locale réglementant l'accès à un territoire et aux ressources qu'il renferme. L'agdal est un territoire strictement délimité. C'est d'abord un concept spatial, géographique et agro-écologique, caractérisé par un milieu physique et des ressources biotiques spécifiques (ressources arborées, pastorales, agricoles...). C'est aussi un corpus de règles d'accès et d'usage qui se rapportent à ce territoire, l'agdal désignant à la fois le territoire, l'institution et les règles. Ces dernières correspondent à l'instauration de mises en défens temporaires définissant l'alternance de périodes d'ouverture et de fermeture du territoire pour des ayants droit bien identifiés. Diverses règles d'usage - définissant les conditions d'exploitation des ressources - complètent souvent le dispositif de gestion (Ilahiane, 1999 ; Araba et al., 2003 ; Auclair et Alifriqui, 2005).

Dans le Haut Atlas, on peut distinguer au moins cinq catégories d'agdals selon la nature des ressources gérées et les fonctions assignées aux mises en défens (Auclair et al., 2005) : les agdals pastoraux, les plus communs, qui représentent d'importantes surfaces en altitude ; les agdals forestiers où les règles d'usages concernent les arbres ; les agdals-vergers visant à protéger la maturation des fruits à certaines périodes de l'année (dans l'arganeraie) ou à limiter la concurrence entre les ayants droit avant la récolte ; les agdals de protection visant à protéger des zones sensibles comme les éboulements sur les villages ou la protection de canaux; enfin, les agdals sanctuaires qui sont des espaces protégés, le plus souvent intégralement, par la seule puissance des interdits qui pèsent sur ces lieux sacrés (cimetière, marabout, source...). 
Jean-Brice Cordier - Didier Genin

Comme le mentionnent Auclair et Alifriqui (2005), l'agdal ne peut être compris qu'au sein de la société qui l'a produit au terme d'une longue confrontation avec un milieu naturel contraignant. Il repose à la fois sur des structures sociales, des pratiques agro-pastorales, des savoirs et croyances et des représentations spécifiques. Longtemps considéré comme une relique des pratiques du passé, l'agdal trouve aujourd'hui une résonance nouvelle avec la généralisation de la rhétorique du développement durable (valorisation des savoirs locaux, "gestion participative" des ressources naturelles...).

Nous nous intéresserons plus particulièrement ici aux espaces forestiers gérés ou non en agdals. Ces espaces ont des fonctions différenciées quant aux ressources mobilisées et aux usages qui leur sont dévolus. Ils contribuent également à la gestion des risques et de l'aléa qui sont une donnée fondamentale du fonctionnement des sociétés montagnardes du Haut Atlas marocain (Garrigues-Cresswell et Lecestre-Rollier, 1987). Ils correspondent ainsi tout à fait aux notions de forêts paysannes, rurales ou domestiques ${ }^{(1)}$, telles qu'elles ont été conceptualisées par Balent (1996) et Michon et al. (2007), en ce sens qu'ils sont pensés et façonnés par des populations locales - en général des agriculteurs au sens large - pour répondre à des modes de vie basés sur une gestion endogène des ressources locales sur le court et le long terme.

Notre objectif est ici de montrer comment ces espaces participent au façonnage de paysages diversifiés et, dans une certaine mesure, à la conservation de milieux forestiers, en mettant en évidence les relations entre pratiques paysannes d'exploitation et conformation des arbres et des peuplements forestiers. Parce que les pratiques d'exploitation des forêts par les populations locales sont le plus souvent conçues de manière à répondre à des besoins de différents ordres et à la valorisation de produits diversifiés, trois échelles d'observation seront abordées : l'arbre, le peuplement forestier et le paysage entendu ici comme " une portion de territoire perçue par un observateur où s'inscrit une combinaison de faits et d'interactions dont on ne voit que le résultat global »(Deffontaines, 1985).

\section{CONTEXTE DE L’ÉTUDE ET MÉTHODOLOGIE}

\section{Les paysages forestiers d'une vallée du Haut Atlas central : les Aît Bouguemez}

Située au cœur du Haut Atlas central entre 1800 et 2200 mètres d'altitude, la vallée des Aït Bouguemez (Province d'Azilal) abrite une trentaine de villages et une population de près de 15000 habitants. Le territoire est enserré entre de puissantes chaînes de montagnes culminant à plus de $3500 \mathrm{~m}$ d'altitude. Il est longtemps resté enclavé et à l'écart des interventions de l'État. La vallée a conservé ainsi des institutions locales vivantes intervenant dans la gestion des ressources renouvelables communes (eau, forêts, pâturages). Le climat y est de type méditerranéen montagnard, avec une variante semi-aride au fond de la vallée et subhumide sur les versants les plus arrosés (Couvreur, 1968). La saison hivernale est froide et longue. Elle présente des épisodes neigeux importants. L'amplitude thermique annuelle est élevée, avec des températures extrêmes qui oscillent entre $-15^{\circ} \mathrm{C}$ et $+45^{\circ} \mathrm{C}$. Les précipitations sont irrégulièrement réparties dans le temps et dans l'espace, mais elles restent abondantes en automne et au printemps (précipitations annuelles variant de 500 à $750 \mathrm{~mm}$ ).

Trois étages de végétation constituent généralement les finages villageois : le fond de vallée qui abrite un territoire cultivé irrigué et des cultures en sec sur les bas de versants les moins

(1) Bien qu'ils puissent parfois présenter des différences sémantiques, nous utiliserons ici ces termes comme synonymes, pour désigner des forêts utilisées et gérées par des populations locales rurales (généralement des agriculteurs au sens large) et les distinguer des autres forêts gérées par les forestiers. 


\section{Lexique}

Agdal : désigne un mode d'appropriation et de gestion de la terre en bien commun mis en œuvre par une institution locale réglementant l'accès à un territoire et aux ressources qu'il renferme. C'est un concept socio-spatial englobant à la fois un territoire, des ressources, des règles et des institutions permettant d'en réguler l'accès et l'usage. Il consiste en l'instauration de mises en défens temporaires définissant l'alternance de périodes d'ouverture et de fermeture du territoire pour des ayants droit bien identifiés, associée à diverses règles d'usage.

Imaa : assemblée coutumière villageoise constituée de tous les chefs de famille et ayant capacité à prendre des décisions concernant la gestion des ressources naturelles et des espaces.

Naib : représentant de la communauté villageoise, faisant le lien entre les Autorités officielles et les membres de la communauté.

Tasaft : Chêne vert (Quercus ilex)

Adghoumam : Genévrier thurifère (Juniperus thurifera)

Tirkki : Genévrier oxycèdre (Juniperus oxycedrus)

escarpés ; les espaces boisés au-dessus des villages, composés essentiellement de trois espèces de Genévriers (Juniperus thurifera, Juniperus oxycedrus, Juniperus phoenicea) ou de Chêne vert (Quercus ilex), et les zones d'altitude au-dessus de $2700 \mathrm{~m}$ constituant des parcours d'estive pour les troupeaux autochtones et transhumants. Nous avons concentré nos études sur la haute vallée appartenant à la fraction tribale des Aït Hakem, qui regroupe dix villages (voir tableau I, p. 574 et figure 1, p. 577 pour la localisation) et dont la surface boisée, tous espaces confondus, représente près de 5000 hectares (Cordier, 2007).

Les systèmes traditionnels de production reposent sur la combinaison de l'agriculture irriguée, relativement intensive sur les terrasses alluviales de fond de vallée, et de l'élevage extensif d'ovins et caprins. Comme dans de nombreux systèmes agraires basés sur la complémentarité verticale en montagne, l'usage combiné des différents étages de végétation est la clé de voûte des systèmes de production, poussant les communautés à définir les conditions d'accès à ces ressources complémentaires. De plus, à la verticalité des étagements de végétation, s'ajoutent des modalités diversifiées d'accès aux ressources associant des appropriations individuelles à différentes formes d'usages collectifs (villageois, intervillageois, intertribaux).

Les zones boisées sont, sur le plan juridique, toutes considérées comme domaniales, mis à part quelques rares exceptions de forêts ou enclaves privées. L'exploitation forestière est officiellement du ressort de l'État depuis le protectorat français. Cependant les populations montagnardes ont toujours refusé de se voir dépossédées de leurs droits sur les espaces boisés et ont perpétué des systèmes locaux d'exploitation des forêts. Dans les conceptions villageoises, deux statuts sont distingués : les agdals forestiers et les zones hors agdal où les règles d'usages sont, mis à part l'identification des ayants droit, inexistantes ou plus lâches. Ces zones hors agdal servent donc de source de bois de feu et de fourrage foliaire pendant une grande partie de l'année. Elles ne font pas l'objet d'une appellation particulière : les villageois les nomment bara $n$ agdal, ce qui signifie " en dehors de l'agdal ». Elles peuvent représenter des surfaces importantes (zone sud de la figure 1) ou avoir été partiellement détruites par suite d'une exploitation intensive (zone nord ; Hammi et al., 2007). Les ayants droit peuvent relever exclusivement d'un village unique ou de plusieurs villages, mais ils sont toujours identifiés. 
La figure 1 (p. 577) montre la répartition des espaces forestiers dans la haute vallée des Aït Bouguemez, en distinguant zones gérées ou non en agdal, et en précisant un accès villageois ou intervillageois.

\section{TABLEAU I Règles de collecte du fourrage foliaire dans les villages \\ de la haute vallée des Aït Bouguemez}

0 : périodes d'ouverture autorisées ; $F$ : fréquence hebdomadaire de collecte autorisée ; Q : quantité de collecte de fourrage foliaire autorisée ; $C$ : compartimentage de l'espace en secteurs ; $E$ : règles sur les espèces végétales à collecter.

\begin{tabular}{|c|c|c|}
\hline Village & Hors agdal forestier & Agdal forestier \\
\hline Rbat n Oufela ........ & $\begin{array}{l}\mathrm{O}: \text { octobre-avril } \\
\mathrm{F}: \text { Collecte }: 2 \mathrm{j} / \text { semaine } \\
\mathrm{Q}: 1 \text { charge (femme ou âne) par } \\
\quad \text { foyer }\end{array}$ & $\begin{array}{l}\mathrm{O}: \text { Uniquement si neige } \\
\mathrm{F}: \text { Tous les jours } \\
\mathrm{Q}: \text { Pas de limitation de quantité }\end{array}$ \\
\hline Akourbi ............ & $\begin{array}{l}\text { O : octobre-mars } \\
\text { F : Pas de restriction } \\
\text { Q : Quantités libres mais } \\
\text { interdiction d'utiliser la mule }\end{array}$ & $\begin{array}{l}\mathrm{O}: \text { Uniquement si neige } \\
\mathrm{F}: \text { Tous les jours } \\
\mathrm{Q}: \text { Quantités autorisées selon nombre } \\
\text { de bêtes }\end{array}$ \\
\hline Ibaqaliun........... & $\begin{array}{l}\text { Superficie réduite } \\
0: \text { Pas de restriction dans les } \\
\quad \text { espaces utilisés. Une zone } \\
\quad \text { mise en défens complètement }\end{array}$ & $\begin{array}{l}\text { O : Uniquement si neige (décision naïb) : } \\
\text { 6-10 jours/an } \\
\text { Q : Quantités libres } \\
\text { Agdal Adazen toujours fermé }\end{array}$ \\
\hline Taghoulid .......... & $\begin{array}{l}\mathrm{O}: \text { Uniquement si neige } \\
\mathrm{F}, \mathrm{Q}: \text { Pas de restriction }\end{array}$ & $\begin{array}{l}\text { O : Uniquement si neige } \\
\mathrm{Q}: \text { Pas de restriction de quantité } \\
\mathrm{C}: \text { Rotation d'ouverture sur } 2 \text { secteurs }\end{array}$ \\
\hline Ifrane ............. & $\begin{array}{l}\mathrm{O}: \text { octobre-mars } \\
\mathrm{Q}: 1 \text { à } 3 \text { charge/famille } \\
\text { en fonction taille du cheptel }\end{array}$ & $\begin{array}{l}\text { O : Uniquement si neige } \\
Q: 1 \text { à } 3 \text { charge/famille en fonction taille } \\
\text { du cheptel } \\
C: 4 \text { secteurs }: 2 \text { utilisés par an }\end{array}$ \\
\hline Aït Ouchi ........... & $\begin{array}{l}0 \text { : octobre-avril } \\
\mathrm{F}, \mathrm{Q}: \text { Pas de restriction } \\
\text { Discussion pour règles de gestion } \\
\text { plus directives }\end{array}$ & $\begin{array}{l}\mathrm{O}: \text { Uniquement si neige } \\
\mathrm{Q}: 1 \text { à } 3 \text { charge/semaine selon taille } \\
\text { du cheptel } \\
\mathrm{C}: \text { 1 seul agdal ouvert par an (sur 2) } \\
\mathrm{E}: \text { parfois uniquement Chêne vert (décision } \\
\text { annuelle de l'assemblée villageoise) }\end{array}$ \\
\hline Ighirine / Iguelwane... & $\begin{array}{l}\mathrm{O}: \text { novembre-mars } \\
\mathrm{F}, \mathrm{Q}: \text { Pas de restriction }\end{array}$ & $\begin{array}{l}\mathrm{O}: \text { Uniquement si neige } \\
\mathrm{C}: 6 \text { secteurs : } 2 \text { utilisés par an } \\
1 \text { jour/semaine/famille } \\
\mathrm{Q}: \begin{array}{c}2-3 \text { charge/famille (décision annuelle } \\
\text { de l'assemblée villageooise) }\end{array} \\
\mathrm{E}: \begin{array}{l}\text { Cueillette autorisée du Chêne vert } \\
\text { uniquement }\end{array}\end{array}$ \\
\hline Aït Ouham.......... & Très peu de disponibilité & $\begin{array}{l}0: \text { Ouverture très rare (pas depuis } 12 \text { ans) } \\
1-10 \text { jours si très fortes chutes de neige }\end{array}$ \\
\hline Zaouit Alemzi ........ & Très peu de disponibilité & $\begin{array}{l}\mathrm{O}: \text { Un seul agdal ouvert (sur 2) si neige } \\
\text { uniquement } \\
\mathrm{Q}: \text { 1 charge/famille }\end{array}$ \\
\hline
\end{tabular}




\section{Méthodologie}

Des entretiens semi-directifs, menés depuis 2004 auprès des responsables des dix villages concernés, les naibs, et différentes personnes-ressources utilisatrices des ressources naturelles (près d'une centaine au total) par une équipe pluridisciplinaire associant sciences de la nature et sciences sociales, ont permis de délimiter précisément les espaces conduits en agdal ainsi que les règles d'usages afférentes. Ces enquêtes ont également permis de cerner les caractéristiques démographiques et socio-productives de chaque village, ainsi que les différents droits d'accès aux espaces porteurs des ressources naturelles locales (agdals pastoraux d'altitude, droits d'eau, espaces forestiers en agdal ou hors agdal, etc.). Ces travaux ont constitué un pool commun d'information, qui a été complété et enrichi au gré des besoins des études particulières sur le territoire. Ils ont été mobilisés ici pour les délimitations des espaces forestiers et les caractérisations de leurs règles et modalités d'usages. Ces données ont constitué la base d'échantillonnage pour l'évaluation écologique et forestière de 27 secteurs soumis à des modalités d'usages contrastées. Ainsi, le choix des secteurs, répartis sur la totalité du territoire étudié, s'est effectué principalement en fonction du statut d'appropriation et de gestion (agdal, hors agdal), de l'espèce arborée principale (Chêne vert tasaft, Genévrier thurifère Adghoumam, combinaison des deux espèces), des usages principaux (fourrage et combustible, bois de construction, autres) et de certaines particularités de gestion (compartimentation de l'agdal avec utilisation en rotation) ou de conformation des peuplements (matorral ${ }^{(2)}$, grands individus).

Un total de 81 placettes ( 3 placettes par secteur) de $400 \mathrm{~m}^{2}$ chacune a été mis en place. Dans chaque placette, des mesures dendrométriques ont été effectuées en ce qui concerne :

- le morphotype de l'arbre : cépée, franc pied ou forme buissonnante sans tronc individualisé ;

- le nombre et le diamètre des tiges ;

- la proportion du feuillage par rapport à la surface du houppier ;

- l'état de l'arbre : vitalité (pourcentage de branches vivantes dans le houppier), traces de pâturage, intensité de coupe ou d'ébranchages (nombre de traces par arbre et par $\mathrm{m}^{2}$ de couvert).

De même, une caractérisation écologique des stations a été effectuée, ainsi que des mesures de recouvrement de la strate herbacée [méthode des points quadrats (Daget et Poissonet, 1972), 3 lignes par placette] et de la diversité floristique (nombre d'espèces par placette).

Ces placettes ont servi aussi à effectuer des échantillonnages d'arbres individuels en relation avec les fonctions qui leur sont attribuées (production de fourrage et de combustible ou production de bois de construction). Ainsi, les données collectées ont été exploitées de manière différentielle selon que l'on s'est référé aux arbres individuels ou aux peuplements (données ramenées à l'hectare). Elles ont fait l'objet d'analyses statistiques par comparaisons de moyennes et analyses de variance.

\section{LES AGDALS FORESTIERS : UNE DIVERSITÉ D’ESPACES ET DE RĖGLES D’USAGES}

Les agdals forestiers se situent à proximité des villages. Ce sont des milieux forestiers ouverts où les espèces arborées sont soit en peuplement pur, soit en mélange. La dominance de telle ou telle espèce varie selon l'exposition, l'altitude, le type de sol et la gestion mise en œuvre.

(2) Formation ligneuse basse (hauteur inférieure à 2 mètres) rencontrée sous climat de type méditerranéen, composée d'arbustes ou de petits arbres, ici des chênes verts en cépées. 
Jean-Brice Cordier - Didier Genin

Dans les strates basses, on retrouve des chaméphytes et quelques espèces herbacées d'intérêt fourrager ${ }^{(3)}$.

Les ressources principalement prélevées dans les agdals forestiers sont le feuillage des arbres qui constitue un aliment de secours et de soudure pour les troupeaux (Genin et al., 2009a). Sur ces territoires ouverts au pâturage toute l'année, la coupe de bois vif et de feuillage est strictement interdite pendant la période de fermeture. L'assemblée des chefs de familles (jmaa) de chaque village décrète l'ouverture de l'agdal en hiver, quand la couverture neigeuse empêche le déplacement des hommes et des troupeaux, et fixe les règles d'usages. Celles-ci résultent à la fois de la coutume et des savoir-faire accumulés au cours des siècles, de négociations et de rapports de force au sein de l'assemblée, et de phénomènes conjoncturels (climat, approvisionnement, etc.). Chaque famille du village est alors autorisée à s'approvisionner en bois de feu et en fourrage foliaire. Chaque village gère un ou deux agdals forestiers de taille variable (entre 20 et 200 ha). D'autre part, il a ou non accès à des zones boisées non gérées en agdal et où s'effectuent les prélèvements de bois de feu et de fourrage foliaire durant la majeure partie de l'année. La disponibilité et les superficies de telles zones boisées conditionnent les règles de gestion mises en œuvre dans les agdals.

Quatre types de règles de collecte du fourrage foliaire peuvent être édictées, lesquelles varient selon les villages (tableau I, p. 574):

- la période d'autorisation de collecte. Il s'agit, en général dans les villages étudiés, des périodes où la neige recouvre le sol. La collecte peut être autorisée tous les jours ou seulement certains jours de la semaine ;

- les quantités de prélèvement autorisées. Différents cas de figure se présentent : utilisation libre sans quotas, quotas en fonction de la taille du troupeau ou quotas fixes indépendamment de la taille du troupeau familial ;

- la division de l'agdal en secteurs (rotation des coupes). C'est par exemple le cas du village d'Ighirine où l'agdal de Ighil-n-Ikis est divisé en 6 secteurs, dont en général deux seulement sont ouverts annuellement à la collecte de feuillage. Ce principe de rotation des coupes de fourrage foliaire est le cas le plus commun dans les agdals de grande taille de la haute vallée des Aït Bouguemez ;

- les espèces à prélever (sélection des essences forestières). Parfois, seuls les prélèvements de Chêne vert sont autorisés tandis que la coupe des genévriers est interdite.

Ce corpus de règles est soumis à des fluctuations en fonction de la taille des agdals disponibles, de l'année climatique, des perceptions vis-à-vis de la ressource et des rapports de force au sein des assemblées villageoises (Lecestre-Rollier, 1986).

Dans les zones forestières périphériques non soumises à l'agdal, les règles sont inexistantes ou plus ou moins lâches : la période d'ouverture fixée par la collectivité locale (en général à partir d'octobre-novembre jusqu'à avril), des jours de collecte peuvent être fixés (village de Rbat : 2 jours par semaine), des quotas institués (village de Rbat : une charge ${ }^{(4)}$ humaine ou de mule par famille; village d'Akourbi : usage des mules interdit; village d'Ifrane : mêmes règles que pour l'agdal).

D’autres produits comme le bois de chauffage (généralement uniquement pour la mosquée) ou les perches de construction peuvent être prélevés dans les agdals forestiers après accord de la jmaa qui en détermine les modalités d'exploitation (quotas, principe de sélectivité, taxes dont le

(3) Les principales espèces rencontrées sont : Thymus pallidus, Calamintha officinalis, Artemisia herba-alba, Rhamnus lyciödes var. atlanticus, Globularia nainii, Pithuranthos scoparius, Berberis hispanica, Crataegus laciniata, Ephedra major ssp. nebrodensis, Euphorbia nicaensis, Euphorbia mesatlantica, Genista scorpius.

(4) Une charge humaine a été évaluée à environ 30 à $35 \mathrm{~kg}$ de biomasse fraîche ; une charge de mule à environ $75 \mathrm{~kg}$. 
(Réalisation J.-B. Cordier, sous ArcGis 8.1)

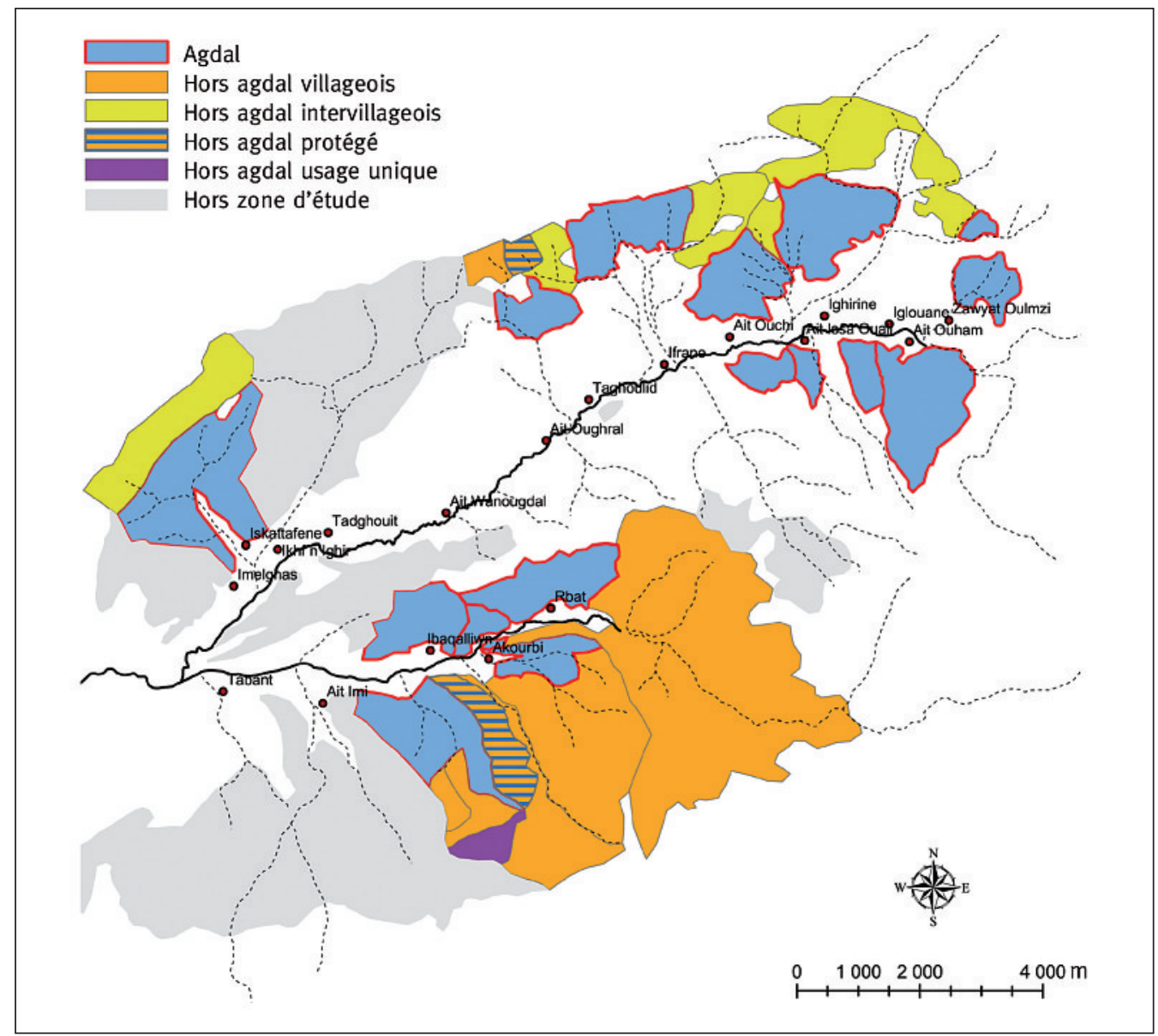

produit alimente la caisse commune du village). Cependant, avec la raréfaction des bois de gros diamètre au cours des dernières décennies, les jmaa semblent de plus en plus réticentes à donner leur accord pour ce type d'exploitation. Dans certains cas, la jmaa attribue à des familles du village (souvent les plus pauvres) des sortes de licences d'exploitation assorties d'un cahier des charges pour la mise en culture de parcelles situées à l'intérieur de l'agdal. Les parcelles cultivées en sec sont livrées à la vaine pâture après la récolte et les arbres restent la propriété commune du village. Au sein de chaque village, des responsables et gardiens sont parfois désignés. Les contrevenants aux règles sont passibles des sanctions décidées par la jmaa, en général une amende qui alimente la caisse commune du village. Les récalcitrants sont éventuellement adressés aux autorités représentant l’État.

Les différentes enquêtes menées au sein des dix villages étudiés ont montré que, en réalité, les populations locales attribuent des fonctions diversifiées à des espaces bien identifiés au sein de leur espace forestier. Certains espaces sont destinés à l'exploitation de ressources particulières et associés à des pratiques différentielles d'exploitation des arbres et des peuplements. 
JeAn-Brice Cordier - Didier Genin

\section{UN FAÇONNAGE DES ARBRES SELON LEURS FONCTIONS D’USAGE : LE CAS DU GENÉVRIER THURIFÈRE}

Une gestion forestière classique se raisonne à l'échelle d'un peuplement qui correspond à une unité de surface homogène à laquelle est attribuée un certain nombre de variables rendant compte de sa valeur économique ou environnementale : le volume de bois à l'hectare, le couvert végétal, etc.

En revanche, la logique d'utilisation en forêt paysanne ne se réfère pas forcément uniquement à la notion de "peuplement", mais aussi à l'arbre en tant qu'unité de gestion. Ceci est illustré dans la dehesa ${ }^{(5)}$ espagnole, où chaque arbre est "conduit" à une forme fonctionnelle productrice de glands, comme on conduirait un peuplement pour la production de bois d'œuvre de qualité par la sylviculture (Montoya, 1996). On trouve ainsi des zones où l'exploitation d'une même espèce forestière est liée à un usage particulier et donc à des pratiques d'exploitation différenciées. II s'ensuit des conformations d'arbres et de peuplement différentes.

Pour illustrer notre propos, nous prendrons le cas du Genévrier thurifère, espèce emblématique du Haut Atlas qui s'élève le plus haut en altitude et qui peut former des grands massifs. Le Genévrier thurifère est le genévrier le plus exploité comme fourrage. Il est réputé pour être d'assez bonne qualité fourragère par rapport aux autres espèces arborées de la zone (Genin et al., 2009a). Son bois constitue un matériau de construction de premier choix dans la zone de par sa solidité et sa capacité à atteindre des brins de taille suffisante pour servir de pièces de charpentes maîtresses. La fonction "production de bois de construction" est associée à une forte régulation du prélèvement de bois de feu et de fourrage foliaire. Cette fonction "bois de construction" disparaît lorsque la priorité est donnée à un prélèvement de fourrage et de bois de feu soutenu.

Nous avons travaillé sur un échantillon de 52 genévriers thurifères présentant des diamètres de tige supérieurs à $20 \mathrm{~cm}$ et répartis, d'une part sur des thuriféraies en agdal réservées à l'usage "bois de construction", et, d'autre part sur des thuriféraies et chênaies mélangées au Genévrier

TABLEAU II

Comparaison de la conformation d'échantillons d'arbres de Genévrier thurifère selon leur usage principal

Les chiffres en gras indiquent des valeurs significativement différentes $(p<0,05)$

\begin{tabular}{|l|c|c|c|c|c|c|c|}
\hline & $\begin{array}{c}\text { Vitalité } \\
\text { du } \\
\text { houppier } \\
(\%)\end{array}$ & $\begin{array}{c}\text { Hauteur } \\
(\mathrm{m})\end{array}$ & $\begin{array}{c}\text { Couvert } \\
\left(\mathrm{m}^{2}\right)\end{array}$ & $\begin{array}{c}\text { Surface } \\
\text { terrière } \\
\left(\mathrm{m}^{2} / \text { arbre }\right)\end{array}$ & $\begin{array}{c}\text { Volume } \\
\text { de } \\
\text { houppier } \\
\left(\mathrm{m}^{3} / \mathrm{ha}\right)\end{array}$ & $\begin{array}{c}\text { Nombre } \\
\text { de coupes } \\
\text { par } \mathrm{m}^{2} \\
\text { de couvert }\end{array}$ & $\begin{array}{c}\text { Volume de } \\
\text { houppier } \\
\text { par } \mathrm{m}^{2} \\
\text { de couvert } \\
\left(\mathrm{m}^{3} / \mathrm{m}^{2}\right)\end{array}$ \\
\hline $\begin{array}{l}\text { Bois de } \\
\text { construction } \\
(\mathrm{N}=34)\end{array}$ & 91,4 & $\mathbf{5 , 4}$ & $\mathbf{4 6 , 2}$ & $\mathbf{0 , 5 4}$ & $\mathbf{1 0 5 , 1}$ & $\mathbf{0 , 3 4}$ & $\mathbf{2 , 1}$ \\
\hline $\begin{array}{l}\text { Fourrage et } \\
\text { combustible } \\
\text { (N = 18) }\end{array}$ & 90,4 & $\mathbf{4 , 0}$ & $\mathbf{2 3 , 1}$ & $\mathbf{0 , 2 9}$ & $\mathbf{3 4 , 1}$ & 0,43 & $\mathbf{1 , 2}$ \\
\hline $\begin{array}{l}\text { Probabilite } \\
\text { du test } \\
\text { de Student }\end{array}$ & $\mathbf{0 , 7 7 6}$ & $\mathbf{0 , 0 0 0}$ & $\mathbf{0 , 0 0 1}$ & $\mathbf{0 , 0 1 8}$ & $\mathbf{0 , 0 0 0}$ & $\mathbf{0 , 2 9 1}$ & $\mathbf{0 , 0 0 0}$ \\
\hline
\end{tabular}

(5) La dehesa correspond à un écosystème anthropique que l'on trouve dans le Sud de l'Espagne, composé d'une forêt claire (5060 arbres/ha) de chênes et soumis au pâturage. Les arbres sont soumis à des tailles destinées à favoriser la production de glands pour l'alimentation animale. 
thurifère uniquement utilisées pour l'approvisionnement en bois de feu et fourrage foliaire (zones en agdal et hors agdal). Des tests de comparaison des moyennes sur les paramètres caractéristiques des arbres (vitalité du houppier, dimensions, indices de pression de coupe et de forme de l'arbre) sont résumés dans le tableau II (p. 578). Les populations locales indiquent qu'elles pratiquent des coupes différenciées selon qu'elles visent à favoriser l'étalement du houppier ou la production de fûts. Elles ont le plus souvent une connaissance précise des arbres en tant qu'individus et du niveau et de la qualité des ressources dont ils sont porteurs.

Les dimensions des arbres exploités pour le bois de construction sont grandes et significativement plus élevées que celles des arbres soumis à une exploitation soutenue pour le bois de feu et le fourrage foliaire $(5,4 \mathrm{~m}$ contre $4,0 \mathrm{~m})$. Les thuriféraies où cette production est associée à une régulation du prélèvement de combustible et de fourrage grâce à la règle de l'agdal, permettent néanmoins de conserver des grands individus à double usage, comme le montre la figure 2. Ceci entraîne des différenciations visibles dans la structure des paysages, dans leur granularité.

FIGURE 2

VERSANT DE LA CHÊNAIE - THURIFÉRAIE D’AKOURBI OÙ UNE PARTIE EST EN AGDAL (à gauche) ET L'AUTRE HORS DE L'AGDAL

Dans le dessin du dessous, ont été matérialisés, à partir d'interprétations des photographies, les genévriers thurifères qui présentent des "empreintes" différenciées selon l'espace considéré (Photo et dessin : J.-B. Cordier)

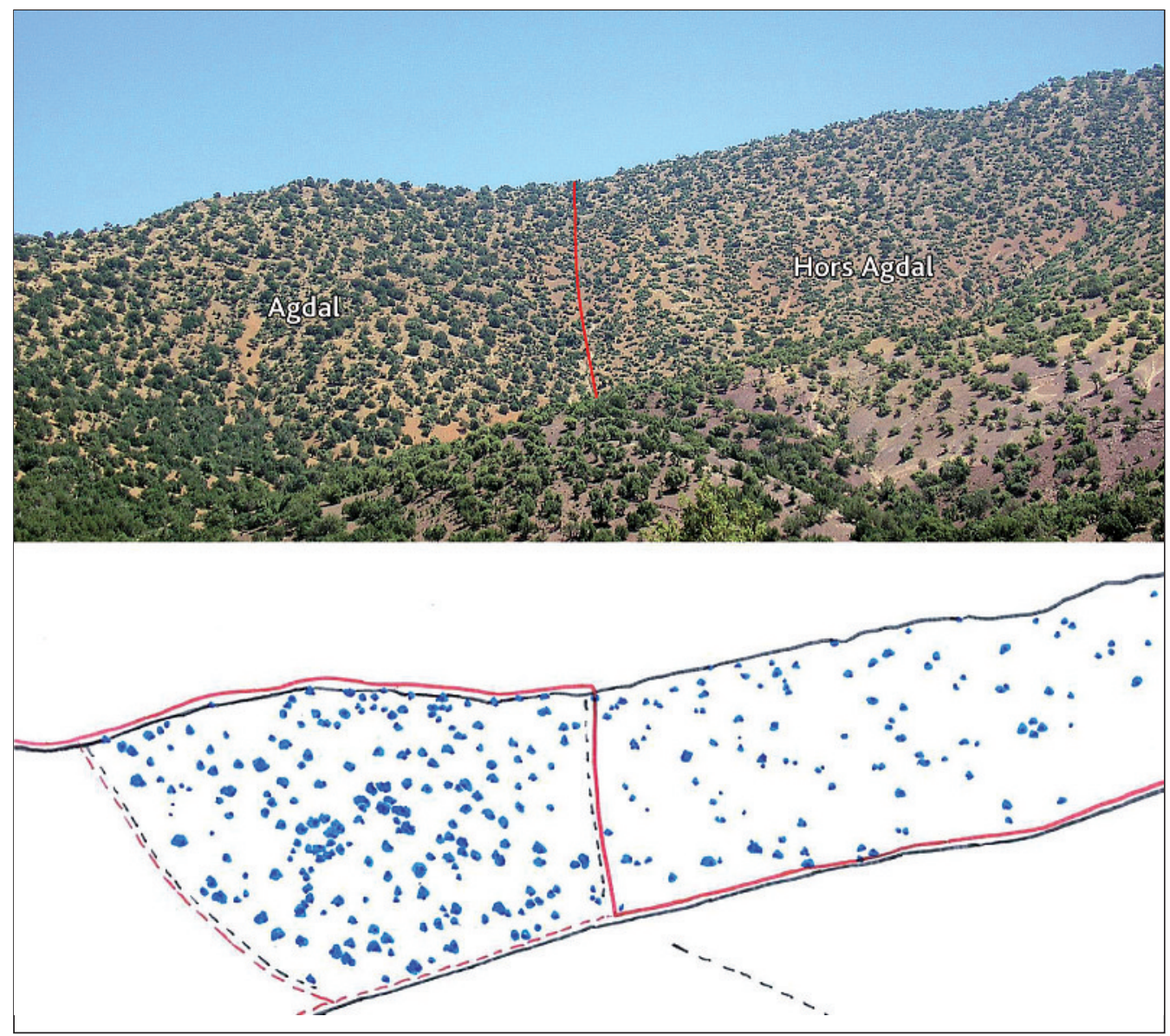


TABLEAU III

Comparaison des moyennes des caractéristiques des arbres soumis à une gestion ou non en agdal

dans des peuplements forestiers à dominante de Chêne vert

de la vallée des Aït Bouguemez (Haut Atlas central, Maroc)

Les chiffres en gras indiquent des valeurs significativement différentes $(p<0,05)$

\begin{tabular}{|l|c|c|c|c|c|c|c|}
\hline & $\begin{array}{c}\text { Couvert } \\
\text { global } \\
(\%)\end{array}$ & $\begin{array}{c}\text { Couvert } \\
\text { du } \\
\text { matorral } \\
\text { de Chêne } \\
\text { vert }(\%)\end{array}$ & $\begin{array}{c}\text { Couvert } \\
\text { de la } \\
\text { strate } \\
\text { herbacée } \\
(\%)\end{array}$ & $\begin{array}{c}\text { Surface } \\
\text { terrière } \\
\text { du Chêne } \\
\text { vert } \\
\left(\mathrm{m}^{2} / \mathrm{ha}\right)\end{array}$ & $\begin{array}{c}\text { Volume du } \\
\text { houppier } \\
\text { du Chêne } \\
\text { vert } \\
\left(\mathrm{m}^{3} / \mathrm{ha}\right)\end{array}$ & $\begin{array}{c}\text { Surface } \\
\text { terrière } \\
\text { des } \\
\text { Genévriers } \\
\left(\mathrm{m}^{2} / \mathrm{ha}\right)\end{array}$ & $\begin{array}{c}\text { Volume } \\
\text { foliaire } \\
\text { des } \\
\text { Genévriers } \\
\left(\mathrm{m}^{3} / \mathrm{ha}\right)\end{array}$ \\
\hline $\begin{array}{l}\text { Hors agdal } \\
\text { (N=22) }\end{array}$ & 25 & $\mathbf{1 3}$ & 11 & 11,3 & $\mathbf{1 6 7 0}$ & 4,7 & 549 \\
\hline $\begin{array}{l}\text { Agdal } \\
\text { (N=35) }\end{array}$ & 26 & $\mathbf{3}$ & 13 & 14,7 & $\mathbf{2 9 9 5}$ & 3,8 & 779 \\
\hline $\begin{array}{l}\text { Probabilité } \\
\text { du test } \\
\text { de Student }\end{array}$ & 0,814 & $\mathbf{0 , 0 0 1}$ & 0,468 & 0,097 & $\mathbf{0 , 0 2 1}$ & 0,634 & 0,389 \\
\hline
\end{tabular}

TABLEAU IV Comparaison des moyennes du nombre des pieds de régénération

(nombre de pieds à l'hectare) du Chêne vert par classe de hauteur et des Genévriers (trois espèces confondues) soumis ou non à une gestion en agdal dans les peuplements forestiers à dominante de Chêne vert de la vallée des Aït Bouguemez (Haut Atlas central, Maroc)

Les chiffres en caractères gras indiquent des valeurs significativement différentes $(p<0,05)$

\begin{tabular}{|l|c|c|c|c|c|c|}
\hline & $\begin{array}{c}\text { Chêne vert } \\
\text { Total }\end{array}$ & $\begin{array}{c}\text { Chêne vert } \\
\mathrm{H}<0,5 \mathrm{~m}\end{array}$ & $\begin{array}{c}\text { Chêne vert } \\
0,5<\mathrm{H}<1,5 \mathrm{~m}\end{array}$ & $\begin{array}{c}\text { Chêne vert } \\
1,5<\mathrm{H}<2,5 \mathrm{~m}\end{array}$ & $\begin{array}{c}\text { Chêne vert } \\
\mathrm{H}>2,5 \mathrm{~m}\end{array}$ & $\begin{array}{c}\text { Genévriers } \\
\text { Total }\end{array}$ \\
\hline $\begin{array}{l}\text { Hors agdal } \\
(\mathrm{N}=22)\end{array}$ & $\mathbf{4 7 8 , 7}$ & 123 & $\mathbf{1 9 4 , 8}$ & $\mathbf{1 3 0 , 2}$ & $\mathbf{9 , 5}$ \\
\hline $\begin{array}{l}\text { Agdal } \\
(\mathrm{N}=35)\end{array}$ & $\mathbf{3 1 6 , 2}$ & 121 & $\mathbf{5 9 , 7}$ & $\mathbf{5 5 , 5}$ & 13,2 & $\mathbf{3 5 , 0}$ \\
\hline $\begin{array}{l}\text { Probabilité } \\
\text { du test } \\
\text { de Student }\end{array}$ & $\mathbf{0 , 0 5}$ & $\mathbf{0 , 9 2 5}$ & $\mathbf{0 , 0 0 5}$ & $\mathbf{0 , 0 1 5}$ & 0,481 & $\mathbf{0 , 0 1 6}$ \\
\hline
\end{tabular}

TABleau V

Comparaison des moyennes des paramètres de structure des peuplements forestiers gérés ou non en agdal Les chiffres en caractères gras indiquent des valeurs significativement différentes $(p<0,05)$

\begin{tabular}{|l|c|c|c|c|c|}
\hline & $\begin{array}{c}\text { Densité de brins } \\
\text { pré-comptables } \\
(3<\varnothing<10 \mathrm{~cm})\end{array}$ & $\begin{array}{c}\text { Surface terrière } \\
\text { du perchis } \\
(10<\varnothing<17 \mathrm{~cm})\end{array}$ & $\begin{array}{c}\text { Surface terrière } \\
\text { des petits bois } \\
(17<\varnothing<25 \mathrm{~cm})\end{array}$ & $\begin{array}{c}\text { Surface terrière } \\
\text { des bois moyens } \\
(25<\varnothing<33 \mathrm{~cm})\end{array}$ & $\begin{array}{c}\text { Surface terrière } \\
\text { des gros bois } \\
(\varnothing>33 \mathrm{~cm})\end{array}$ \\
\hline $\begin{array}{l}\text { Hors agdal } \\
(\mathrm{N}=22)\end{array}$ & $\mathbf{2 4 4}$ & $\mathbf{1 , 7}$ & 1,9 & 3,4 & 3,2 \\
\hline $\begin{array}{l}\text { Agdal } \\
(\mathrm{N}=35)\end{array}$ & $\mathbf{5 2 5}$ & $\mathbf{4 , 2}$ & 2,6 & 5,4 & 2,7 \\
\hline $\begin{array}{l}\text { Probabilité } \\
\text { du test } \\
\text { de Student }\end{array}$ & $\mathbf{0 , 0 0 3}$ & $\mathbf{0 , 0 1 0}$ & 0,243 & 0,170 & 0,907 \\
\hline
\end{tabular}


Le croquis de la figure 2 montre ainsi "l'empreinte" spatiale plus importante des individus de Genévrier thurifère dans la zone conduite en agdal. Les pratiques d'exploitation des arbres mises en œuvre participent ainsi à la conformation de faciès plus forestiers à proximité des villages, plus à même de satisfaire aux besoins alimentaires des troupeaux lors d'épisodes neigeux.

\section{CARACTÉRISATION DE L'INCIDENCE DES PRATIQUES D'AGDAL SUR LA STRUCTURE DES PEUPLEMENTS}

Dans de tels écosystèmes, il est possible d'observer une forte variabilité morphologique, que ce soit à l'échelle de l'individu (arbre) ou à l'échelle de la physionomie du peuplement dans son ensemble. Cette physionomie structurale des arbres et des peuplements traduit les types d'usages et les fonctions des ressources arborées mobilisées par les populations locales.

Une étude comparative a ainsi été conduite sur 57 placettes de $400 \mathrm{~m}^{2}$ représentatives de peuplements de Chêne vert dominant - 35 en agdal et 22 hors agdal - afin de caractériser l'impact de ces deux modes de gestion sur les caractéristiques dendrométriques des arbres.

Il apparaît que, bien qu'ayant des recouvrements totaux équivalents, l'agdal et le hors agdal ne contiennent pas le même type de ressources (tableau III, p. 580).

La fonction de réserve alimentaire de l'agdal pour les troupeaux s'exprime par le fait qu'il contient plus de fourrage foliaire de Chêne vert par unité de surface (2 $995 \mathrm{~m}$ 3/ha contre $1670 \mathrm{~m}^{3} / \mathrm{ha}$ ). En revanche, la différence de surface terrière de Chêne vert n'est pas significative $\left(14,7 \mathrm{~m}^{2} /\right.$ ha contre $\left.11,3 \mathrm{~m}^{2} / \mathrm{ha}\right)$. Le couvert herbacé n'est pas influencé par le type de gestion (en général d'un recouvrement inférieur à $30 \%$ ), mais la qualité du parcours pâturable directement par les troupeaux est supérieure hors de l'agdal car il englobe des zones de matorral dont les chênes verts sont également très pâturés. Ce type de formation, souvent considérée comme dégradée, remplit ici des fonctions fourragères clairement mentionnées par les populations locales. Il a, de plus, une fonction protectrice du sol de par son recouvrement.

Les ressources en bois et en fourrage des genévriers ne sont pas significativement différentes dans et hors de l'agdal dans les zones dominées par le Chêne vert.

En ce qui concerne la régénération (diamètre des pieds inférieur à $10 \mathrm{~cm}$ ), on observe un nombre de pieds par hectare plus important hors agdal pour le Chêne vert (478,7 pieds/ha contre 316,2), étant donné encore l'importance des formations de matorral (tableau IV, p. 580). Les classes de hauteur situées en 0,5 et 2,5 m sont logiquement plus représentées, car elles correspondent aux hauteurs moyennes du matorral. En revanche, il est intéressant de constater que le nombre moyen de régénérations "naissantes" (moins de $50 \mathrm{~cm}$ ) n'est pas différent d'un espace à l'autre (123 contre 121). C'est également le cas de la régénération supérieure à 2,5 mètres. La différence entre les classes « entre 1,5 et 2,5 $\mathrm{m}$ » et " plus de $2,5 \mathrm{~m}$ » est d'autant plus flagrante hors de l'agdal. Il y a donc un arrêt dans la dynamique de la régénération, qui ne peut se développer en hauteur à cause de l'ébranchage.

Concernant la structure (au sens dendrométrique) des peuplements, les seules différences entre l'agdal et le hors agdal concernent la densité et la surface terrière du perchis (diamètre des brins entre 10 et $17 \mathrm{~cm}$ ) (tableau V, p. 580). En revanche, le matériel sur pied des autres classes de diamètre n'est pas significativement différent. Cela peut s'expliquer par le fait que les brins de faible diamètre sont exploités en priorité hors de l'agdal pour un usage quotidien de bois de feu car ils sont plus faciles à abattre. Les restrictions d'usages dans l'agdal permettraient alors de protéger les jeunes brins. 
JeAn-Brice Cordier - Didier Genin

Les dynamiques des peuplements de Chêne vert apparaissent ainsi bien différentes dans et hors de l'agdal (tableaux IV et V, p. 580) :

- d'un côté, l'agdal témoigne d'un relatif équilibre entre les différentes classes de hauteurs de la régénération, ainsi que d'un nombre important de brins de petit diamètre : il s'agit d'un peuplement apte à se renouveler ;

- d'un autre côté, le hors agdal possède un plus grand nombre d'unités de régénérations de Chêne vert, mais sa capacité d'affranchissement dans la classe "perchis" s'amenuise au fur et à mesure des exploitations des brins du matorral. Les peuplements arborescents sont surtout constitués de vieux arbres dont les houppiers sont surexploités.

D'autres impacts des modalités des pratiques d'exploitation des arbres sur l'état des ressources et la structure des peuplements ont été mis en évidence par Cordier (2007), notamment en ce qui concerne la mise en place de rotations pluriannuelles d'exploitation dans certains agdals. En effet, on observe des caractéristiques structurales des individus de Chêne vert plus importantes dans les agdals présentant des rotations pluriannuelles de coupe que dans les agdals où l'utilisation n'est pas compartimentée (surface terrière de 19,8 $\mathrm{m}^{2} /$ ha contre $11,1 \mathrm{~m}^{2} /$ ha ; volume du houppier de $3956 \mathrm{~m}^{3} / \mathrm{ha}$ contre $2453 \mathrm{~m} 3 / \mathrm{ha}$ ). Ces valeurs reflètent la présence d'arbres plus développés qui marquent aussi les paysages forestiers de la haute vallée des Aït Bouguemez.

\section{UNE DIVERSIFICATION PHYSIONOMIQUE DES MILIEUX FORESTIERS EN FONCTION DES PRATIQUES MISES EN GUVRE : L'EXEMPLE DU VILLAGE D'IBAQALIUN}

En regardant avec un œil averti les paysages forestiers de la haute vallée des Aït Bouguemez, on s'aperçoit que ceux-ci sont formés d'une mosaïque de milieux. Dans le village d'Ibaqaliun par exemple, l'espace forestier est compartimenté en différentes zones que l'on peut individualiser physionomiquement et dont on peut retracer l'histoire (figure 3, p. 583).

Sur le versant orienté au sud, à proximité immédiate du village, se trouve l'agdal d'Adazen. II est constitué uniquement de genévriers de Phénicie (Juniperus phoenicea), arbres au feuillage non palatable pour les animaux, généralement multicaules et produisant des perches de faible diamètre utilisées comme supports transversaux aux poutres dans la fabrication des toitures. Aux dires d'un responsable du village, cet espace a été exploité pour la dernière fois il y a 10 ans, pour la construction d'une mosquée. Depuis, aucune coupe n'aurait été réalisée. Il s'agit d'une formation arborée ouverte en relativement bon état en ce qui concerne les arbres. Elle est intensément pâturée.

Sur le versant orienté au nord du territoire villageois se trouve la majeure partie de l'espace forestier et de l'espace de parcours.

L'agdal forestier est constitué de quatre compartiments :

- Imla : le plus fort de la pente, jusqu'à la crête limite avec le territoire d’Aït Imi ;

- Ouftis : entre la pente forte et la plaine ;

- Loutta : la plaine ;

- Assamer : le versant ensoleillé en dessous de la falaise. Ce compartiment est divisé en deux parties, la première (constituée de genévriers) étant réservée à la coupe de bois pour chauffer la mosquée pendant l'hiver ainsi que l'eau des ablutions, la seconde est constituée principalement de chênes verts.

Cet agdal est ouvert en hiver pour permettre la récolte de bois pour le chauffage des habitations et pour la cuisine, et de fourrage foliaire pour alimenter les animaux. La période d'ouverture peut 


\section{FigurE 3 LES FONCTIONS DIFFÉRENCIÉES DES ESPACES BOISÉS DANS LE VILLAGE D’IBAQALIUN (vallée des Aït Bouguemez, Haut Atlas central, Maroc) \\ Image : (C) SPOT IMAGE / HOA-QUI / EYEDEA}

En violet : agdal d’Adazen réservé à l'exploitation de perches (espèce dominante : Genévrier de Phénicie). En bleu : agdal utilisé pour le fourrage foliaire (espèces dominantes : Chêne vert et Genévriers), divisé en 4 compartiments. En vert, partie de l'agdal réservée uniquement à la coupe de bois de feu pour la mosquée (espèces dominantes : Genévriers). En jaune : zone hors de l'agdal actuellement ouverte à la coupe (espèces dominantes : Chêne vert et Genévriers). En rouge, zone hors de l'agdal où toute coupe est actuellement interdite (espèces dominantes : Chêne vert et Genévriers). En marron, zone hors de l'agdal réservée à l'exploitation de poutres (espèce dominante : Genévrier thurifère). En beige : zones hors de l'agdal éloignées du village ou peu accessibles et non règlementées (espèces dominantes : Chêne vert et Genévriers).

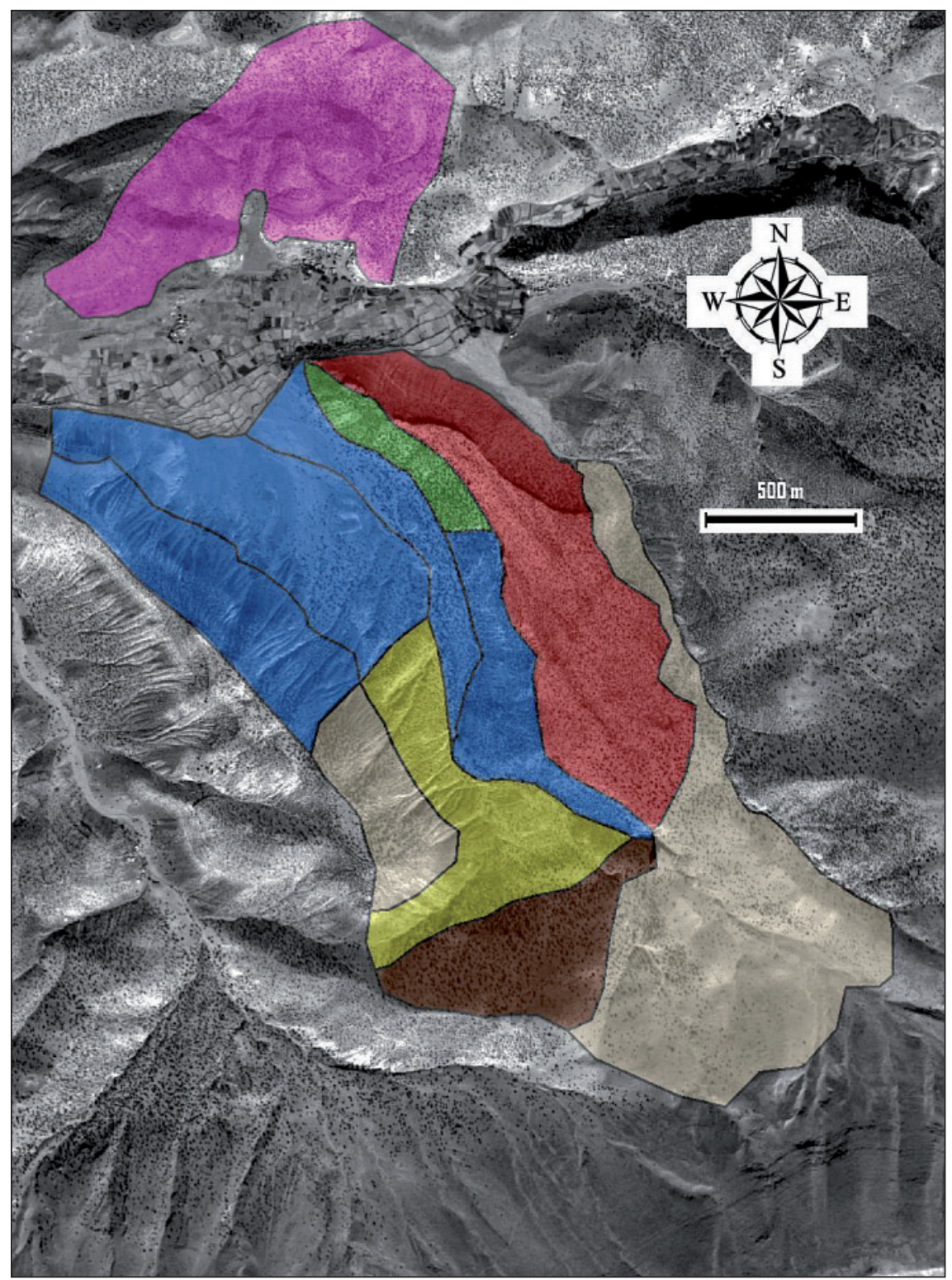


Jean-Brice Cordier - Didier Genin

atteindre plusieurs mois, contrairement aux agdals d'autres villages où cette période est beaucoup plus limitée, car les habitants d'Ibaqaliun ont peu accès à des zones forestières d'usage commun.

L'espèce forestière la plus exploitée pour le fourrage est le Chêne vert, car, selon les locaux, " il se régénère plus vite que le genévrier". Afin de laisser repousser le Chêne vert de l'agdal, les villageois ne l'exploitent pas en moyenne pendant trois ans. Cela implique un système de coupes par rotations entre les différents compartiments. Les compartiments Ouftis et Imla sont toujours ouverts ensemble afin de procurer une ressource suffisante. Si le compartiment Loutta possède la plus petite surface en chênes verts, il n'est pas certain que la ressource foliaire y soit moindre, car cet espace est constitué de grands chênes ainsi que de grands genévriers thurifères.

Dans la zone hors agdal, dont l'utilisation des ressources arborées est exclusive du village, l'exploitation se faisait jusqu'à une période récente de manière libre. Cependant, les habitants d'Ibaqaliun ont remarqué une dégradation de la forêt et une perte d'intérêt fourrager de la strate arborée. Face à cette dégradation de l'espace hors agdal, les villageois ont mis en place il y a 9 ans un système de rotation afin de laisser se régénérer une zone pendant que l'autre est exploitée. On a donc deux compartiments bénéficiant de ce régime de protection. Les zones les plus éloignées ou les moins accessibles ne sont pas incluses dans ce système car " les gens y coupent peu ". Le temps de protection est de 4 ans. Cet espace est donc conduit comme un agdal sur un pas d'exploitation pluriannuel. Dans cette zone hors agdal, un versant entier, constitué de grands genévriers thurifères est réservé exclusivement à l'exploitation des poutres, " car on y trouve encore des gros arbres".

Ces formations, d'une part, participent à la diversité écosystémique qui est un des éléments de la biodiversité ${ }^{(6)}$, et, d'autre part, représentent de véritables espaces-ressources intégrés ayant des fonctions complémentaires. Dans le cas du village d'Ibaqaliun, comme dans les autres d'ailleurs, le grain des paysages forestiers varie ainsi en relation avec les fonctions que ces derniers assurent au sein de la société locale qui les exploite. Il en résulte une mosaïque d'espaces d'une grande complexité.

\section{DISCUSSION ET CONCLUSION}

\section{De l'arbre au peuplement et au paysage : des niveaux d'échelles complémentaires pour une écologie des paysages forestiers du Haut Atlas}

Les forêts étudiées ici sont des forêts claires dont le recouvrement est modeste en regard des forêts classiques et où la distance entre les arbres permet une certaine hétérogénéité dans les formes individuelles de croissance. Les arbres qui s'y développent, nous l'avons vu, voient leur port modifié en fonction des rôles que leur attribuent les populations locales. L'arbre est ainsi individualisé, à la fois dans les représentations des gens - qui peuvent bien souvent identifier des individus d'arbres pour illustrer la description de leurs actions - et dans les pratiques qui s'y rapportent (conduite pour la collecte de fourrage et de combustible, ou pour la collecte de poutres ou de perches). La description des arbres est donc ici un indicateur intéressant pour, d'une part, évaluer la granularité et la structure d'un peuplement forestier et, d'autre part, en comprendre ses fonctions. L'échelle du peuplement permet une inscription spatiale des arbres. C'est l'échelle classique et primordiale du forestier, qui donne accès à une description globale de la formation arborée et à une évaluation du niveau de ressources disponibles et utilisées.

(6) Il est habituellement admis que la biodiversité englobe trois composantes : la diversité génétique (diversité des gènes des différents organismes), la diversité spécifique (diversité des espèces animales et végétales) et la diversité écosystémique (l'écosystème étant un système en interactions composé de l'ensemble des populations occupant un territoire et des éléments abiotiques qui lui sont liés, formant ainsi une unité fonctionnelle). 
L'échelle du paysage est un moyen de mieux comprendre la structuration du territoire et d'avoir une vision d'ensemble de la répartition et des interactions des portions d'espaces forestiers dans les finages complexes que constituent les vallées montagnardes du Haut Atlas. Cette échelle est intéressante pour appréhender comment est aménagée, voire "façonnée" la forêt afin de répondre à des besoins et des modes de vie d'une population humaine très ancrée dans son territoire (Burel et Baudry, 1999). L'exemple pris du village d'Ibaqaliun en est une illustration, dans laquelle on peut matérialiser les tâches formées dans l'espace forestier villageois et leurs fonctions de pourvoyeuses de ressources diversifiées. Les interactions et complémentarités avec les autres portions du territoire (zones de cultures irriguées, villages, espaces de parcours d'altitude) sont aussi clairement matérialisées à cette échelle.

\section{L’agdal forestier comme archétype de la forêt rurale}

En développant le concept de forêt domestique, Michon et al. (2007) ont montré qu'il n'existe pas qu'une forme monolithique qui permette d'appréhender la forêt et sa gestion. La forêt domestique est une forme particulière de gestion forestière destinée à répondre à des besoins diversifiés des populations locales qui se développent dedans ou à proximité. C'est un lieu où se rencontrent des modes de vie locaux, une culture et des relations socio-politiques à la fois au sein du groupe local qui l'exploite et avec l'extérieur. Pour reprendre l'expression de ces auteurs, c'est " une forêt à vivre qui intègre production et conservation au sein des dimensions sociales, politiques et spirituelles " de la société qui l'utilise. Les pratiques d'exploitation et de gestion qui en découlent, d'une part, répondent aussi à des objectifs et des rationalités propres qu'il y a lieu de mieux comprendre, et, d'autre part, ont des impacts sur les composants physiques et physionomiques de la forêt. Les pratiques locales façonnent la création de milieux forestiers contrastés favorisant un paysage diversifié et une multifonctionnalité des ressources arborées. Cette multifonctionnalité est bien souvent une stratégie d'optimisation de l'utilisation des ressources, guidée par des objectifs économiques et par la nécessité de répondre à des besoins dont les opportunités de couverture sont bien souvent difficiles à trouver en dehors de la valorisation des ressources locales.

Ainsi, en dépit de ses apparences de "forêt dégradée », " mal gérée », ou " peu rentable », la forêt rurale est porteuse de qualités du développement durable (Nasi et Michon, 2006). Elle n'est pas un modèle bien huilé, que ce soit en termes de sanctuaire de biodiversité, de concertation et de redistribution des bénéfices, ou de production de bois, mais elle est porteuse de qualités partielles dans tous ces domaines (Asbjornsen et al., 2004). Elle permet de plus d'intégrer d'autres dimensions fondamentales dans la gestion de la forêt, comme la gouvernance et la patrimonialisation des ressources et des milieux (Verdeaux, 1999).

Les agdals forestiers du Haut Atlas représentent une forme originale de gestion de forêt rurale. Ils constituent ainsi des espaces-ressources intégrés dans une gestion dans l'espace et dans le temps des ressources. Ils permettent, sur le temps long, de réagir à des aléas prévisibles mais non bornés dans le temps et, dans le cycle annuel, d'avoir un accès sécurisé et pérenne à des ressources sur des séquences de temps bien identifiées. Les agdals permettent indéniablement une certaine protection de la forêt, mais ils doivent être resitués au sein de l'espace forestier utilisé dans sa globalité par les populations locales.

\section{Gestion paysanne des espaces forestiers du Haut Atlas, quel avenir ?}

Différents auteurs ont montré que la forêt du Haut Atlas est sous pression environnementale (Alifriqui, 1993 ; Aderdar, 2000). Dans la haute vallée des Aït Bouguemez, Hammi et al. (2007) ont montré qu'en quarante ans la superficie boisée s'est réduite de $20 \%$. Ce chiffre est à mettre 
en relation avec un presque doublement de la population humaine sur cette période. Les besoins, notamment en bois de chauffage et en fourrage foliaire, ont explosé. Hammi et al. (2007) ont cependant montré que la gestion de type agdal a permis un maintien du couvert boisé dans ces espaces, avec des délimitations très stables et souvent des augmentations de recouvrement, mais des problèmes de transfert de pression sur les zones boisées hors agdal apparaissent alors probables. L'agdal forestier correspond plus à une volonté de sécuriser l'accès pérenne aux ressources arborées à proximité des villages qu'à une gestion conservatrice de la forêt dans son ensemble. Toutefois, nous l'avons vu, il constitue un système de gestion qui présente une utilité évidente pour les populations locales et qui favorise une diversité de paysages intéressante. Il ne faut cependant pas minimiser les menaces réelles et les contraintes qui pèsent sur ces modes de gestion traditionnels dans un contexte de mutations rapides touchant les systèmes de production, l'économie régionale et les institutions locales. Si ces pratiques ne semblent pas menacées à court terme vu l'intérêt que leur prête la grande majorité des usagers, des tendances lourdes voient le jour (individualisation des unités de production, perte de dynamisme de l'élevage extensif, relations socio-économiques accrues avec l'extérieur). De plus, dans un contexte de forte pression sur les ressources et de différenciation sociale accrue, la discipline collective en termes de gestion des ressources est de plus en plus difficile à mettre en œuvre.

Dans certains cas, l'agdal apparaît comme une institution en crise, avec le désintérêt des jeunes générations et la difficile transmission des savoirs locaux. Mais il est bien difficile de tirer une conclusion définitive sur le devenir des agdals. Dans de nombreuses régions du Haut Atlas, les pratiques d'agdal sont vivantes, avec l'adaptation permanente des règles et parfois même la création d'agdal répondant à de nouveaux besoins ou à de nouvelles contraintes. Dans certains cas, les assemblées villageoises maintiennent leur agdal ou le réactivent, évoquant les ancêtres et la tradition, alors que l'importance économique des ressources en jeu semble dérisoire. L'agdal, qui constitue un outil utile pour une gestion durable des ressources, serait-il aussi un moyen, parmi d'autres, de conforter l'identité et la cohésion du groupe alors que les forces centrifuges se font de plus en plus pressantes?

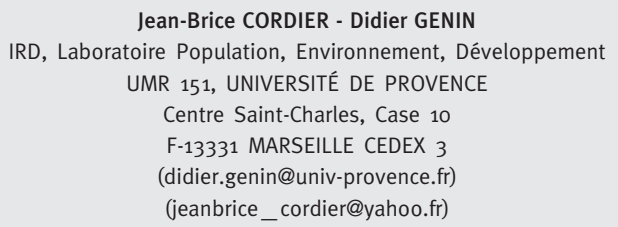

Cette étude s'inscrit dans le cadre du programme POPULAR « Politiques publiques et gestions paysannes de l'arbre et de la forêt : alliance durable ou dialogue de dupes ? " qui a reçu le soutien financier de l'Agence nationale de la recherche (ANR-06-PADD-014).

\section{BIBLIOGRAPHIE}

ADERDAR (M.). - Espaces forestiers et aménagement des zones de montagne : le cas du Haut Atlas de Marrakech. - Grenoble : Université Joseph Fourier, 2000. - 367 p. (Thèse de Doctorat).

ALIFRIQUI (M.). - La Dégradation du couvert végétal dans le Haut Atlas de Marrakech : causes et conséquences. In : Montagnes et hauts pays de l'Afrique : utilisation et conservation des ressources / A. Bencherifa, éditeur. - Publication Faculté Lettres \& Sciences humaines, Université Mohamed V, n 29, 1993, pp. 319-329. 
ARABA (A.), BOURARACH (K.), CHICHE (J.), YESSEF (M.). - Recueil des pratiques et savoirs locaux en matière de transhumance et conduite du cheptel. - Rabat : Institut agronomique et vétérinaire Hassan II, 2003. - $99 \mathrm{p}$.

ASBJORNSEN (H.), ANGELSEN (A.), BERCHER (B.), MICHON (G.), RUIZ PREZ (M.), WIJESEKARA (V.P.R.), Editors. - Cultivating (in) Tropical Forests ? - Lofoten (Norway) : European Tropical Forest Research Network Publication Series, 2004. - 189 p.

AUCLAIR (L.), ALIFRIQUI (M.). - Les agdals du Haut Atlas marocain. Enjeux d'une recherche pluridisciplinaire. - Rabat : Centre Jacques Berque (CJB). - Cahiers de Recherche, $\mathrm{n}^{\circ}$ 3, 2005, pp. 60-79.

AUCLAIR (L.), GENIN (D.), HAMMI (S.), KERAUTRET (L.). - Les agdals du Haut Atlas marocain : les effets d'un mode de gestion traditionnel sur les ressources sylvopastorales. In : Actes du Séminaire international "Le développement durable des zones de montagne », Association marocaine d’Agro-économie (AMAECO), Rabat, 1-2/12/2005. - 20 p.

BALENT (G.). - La Forêt paysanne dans l'espace rural. Biodiversité, paysages, produits. - Versailles : Institut national de la Recherche agronomique, 1996. - 276 p. (Études et recherches sur les systèmes agraires et le développement ; 29).

BUREL (F.), BAUDRY (J.). - Écologie du paysage : concepts, méthodes et applications. - Paris : Tec \& Doc, 1999. $-362 \mathrm{p}$.

CORDIER (J.-B.). - Impacts écologiques des pratiques d'agdal sur les peuplements forestiers et propositions de gestions alternatives. - Nancy : ENGREF, 2007. - 88 p. (Mémoire FIF).

COUVREUR (G.). - La Vie pastorale dans le Haut Atlas central. - Revue de Géographie du Maroc, 13, 1968, pp. 3-47.

DAGET (Ph.), POISSONET (J.). - Un procédé d'estimation de la valeur pastorale des pâturages. - Fourrages, 49, 1972, pp. 31-39.

DEFFONTAINES (J.-P.). - Étude de l'activité agricole et analyse du paysage. - L'espace géographique, vol. $14, \mathrm{n}^{\circ} 1,1985$, pp. 37-47.

GARRIGUES-CRESSWELL (M.), LECESTRE-ROLLIER (B.). - Gérer les aléas. Les sociétés du Haut Atlas marocain. - Techniques \& Cultures, 38, 1987, pp. 69-95.

GAUQUELIN (T.), BERTAUDIERE (V.), MONTES (N.), BADRI (W.), ASMODE (J.F.). - Endangered stands of thuriferous juniper in the western Mediterranean basin: ecological status, conservation and management. Biodiversity \& Conservation, 8, 2000, pp. 1476-1498.

GENIN (D.), FOUILLERON (B.), KERAUTRET (L.). - Un tempo bien tempéré... Place et rôles des agdals dans les systèmes d'élevage de la vallée des Aït Bouguemez (Haut Atlas central). In : Les agdals de l'Atlas marocain : savoirs locaux, droits d'accès, gestion de la biodiversité / L. Auclair, M. Alifriqui. - Rabat : IRD-UCAM-IRCAM, 2009a (sous presse).

GENIN (D.), KERAUTRET (L.), HAMMI (S.), CORDIER (J.-B.), ALIFRIQUI (M.). - Biodiversité et pratiques d'agdal : un élément de l'environnement à l'épreuve de ses fonctions d'utilité pour les sociétés locales du Haut Atlas central. In : Les agdals de l'Atlas marocain : savoirs locaux, droits d'accès, gestion de la biodiversité / L. Auclair, M. Alifriqui. - Rabat : IRD-UCAM-IRCAM, 2009b (sous presse).

HAMMI (S.), SIMONNEAUX (V.), ALIFRIQUI (M.), AUCLAIR (L.), MONTES (N.). - Étude sur le long terme de la dynamique forestière dans la haute vallée des Aït Bouguemez (Haut Atlas central) : impact des modes de gestion. - Sécheresse, vol. 18, $\mathrm{n}^{\circ} 4$, 2007, pp. 271-277.

ILAHIANE (H.). - The berber agdal institution: indigenous range management in the Atlas mountains. Ethnology, vol. 38, $\mathrm{n}^{\circ}$ 1, 1999, pp. 21-45.

LECESTRE-ROLLIER (B.). - L’Espace collectif et les conflits chez les Aït Bouguemez (Haut Atlas marocain). Techniques et culture, $\mathrm{n}^{\circ} 7,1986$, pp. 95-111.

MICHON (G.), de FORESTA (H.), LEVANG (P.), VERDEAUX (F.). - Domestic forests: a new paradigm for integrating local communities' forestry into tropical forest science. - Ecology \& Society, vol. 12, $\mathrm{n}^{\circ} 2,2007$, [online].

MONTOYA (J.M.). - La poda de los arboles forestales. - Agroguias Mundi Prensa, 1996. - 87 p.

NASI (R.), MICHON (G.), Coord. - POPULAR : Politiques publiques et gestion paysanne de l'arbre et de la forêt : alliance durable ou dialogue de dupes ? Proposition de recherche du Programme fédérateur " Agriculture et développement durable ». - Paris : ANR, 2006. - 49 p.

VERDEAUX (F.), éditeur. - La Forêt-monde en question : recomposition des rapports des sociétés à la forêt dans les pays du Sud. - Autrepart, vol. 9, 1999, 182 p. 


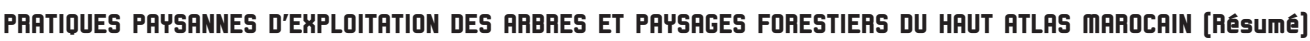

Dans le Haut Atlas marocain, les sociétés rurales berbères ont mis en place des systèmes de gestion endogènes qui ont permis de maintenir la disponibilité des ressources forestières et pastorales sur le temps long, notamment par la pratique de l'agdal. Celle-ci correspond à l'instauration de mises en défens temporaires sur des territoires bien définis et l'élaboration de règles d'usages pour des ayants droit bien identifiés. Les modalités des règles et des pratiques d'usages qui en découlent ont des incidences directes sur les formes des arbres et la structure des peuplements forestiers. On assiste ainsi à un façonnage de paysages diversifiés au sein d'une forêt rurale qui répond à des modes de vie et des besoins qui intègrent production, conservation et gestion des aléas d'un milieu montagnard contraignant au sein de dimensions sociales, politiques et spirituelles propres aux sociétés locales qui les ont pensées.

\section{FARIIERS' LOGGING PRACTICES AND FOREST LANDSCRPES IN THE UPPER RTLAS, mOROCCO [Abstract]}

In the Moroccan Upper Atlas, the rural Berber societies have established endogenous management systems that maintain the availability of forest and pastoral resources in the long term, in particular through the practice of agdal. This consists in temporarily setting aside well-defined areas of grazing land and establishing rules of usage for clearly identified eligible users. The rules devised and the resulting usage have a direct impact on tree shapes and the structure of forest stands. In this way, diversified landscapes are formed in rural forests that match the lifestyles and needs of the population, through the integration of production, conservation and management of unexpected events in a demanding upland environment against the social, political and spiritual background that is inherent to the local societies that devised these systems. 